\title{
Absence of Wolbachia endobacteria in the human parasitic nematode Dracunculus medinensis and two related Dracunculus species infecting wildlife
}

Jeremy M Foster ${ }^{1 *}$, Frédéric Landmann², Louise Ford ${ }^{3}$, Kelly L Johnston ${ }^{3}$, Sarah C Elsasser ${ }^{4}$, Albrecht I Schulte-Hostedde ${ }^{4}$, Mark J Taylor ${ }^{3}$ and Barton E Slatko ${ }^{1}$

\begin{abstract}
Background: Wolbachia endosymbionts are a proven target for control of human disease caused by filarial nematodes. However, little is known about the occurrence of Wolbachia in taxa closely related to the superfamily Filarioidea. Our study addressed the status of Wolbachia presence in members of the superfamily Dracunculoidea by screening the human parasite Dracunculus medinensis and related species from wildlife for Wolbachia.

Findings: $D$. medinensis, D. lutrae and D. insignis specimens were all negative for Wolbachia colonization by PCR screening for the Wolbachia ftsZ, 165 rRNA and Wolbachia surface protein (wsp) sequences. The quality and purity of the DNA preparations was confirmed by amplification of nematode $18 \mathrm{~S}$ rRNA and cytochrome c oxidase subunit I sequences. Furthermore, Wolbachia endobacteria were not detected by whole mount fluorescence staining, or by immunohistochemistry using a Wolbachia-specific antiserum. In contrast, positive control Brugia malayi worms were shown to harbour Wolbachia by PCR, fluorescence staining and immunohistochemistry.

Conclusions: Three examined species of Dracunculus showed no evidence of Wolbachia endobacteria. This supports that members of the superfamily Dracunculoidea are free of Wolbachia. Within the order Spirurida, these endosymbionts appear restricted to the Filarioidea.
\end{abstract}

Keywords: Dracunculus, Wolbachia, Guinea worm

\section{Findings}

With the exception of Loa loa, all examined filarial nematodes that infect humans as their definitive host contain Wolbachia. These obligate intracellular symbionts have emerged as a novel target for filarial disease control [1-3]. Filarial species containing Wolbachia predominantly fall within the subfamilies Onchocercinae and Dirofilariinae of the family Onchocercidae, although Madathamugadia hiepei (subfamily Splendidofilariinae) is also colonized [4]. As a result, there has been extensive screening for Wolbachia in these taxa leading to the hypothesis that ancestral acquisition of Wolbachia occurred in the lineage leading to these subfamilies [5-7]. Screening for Wolbachia presence in phylogenetically divergent nematode species

\footnotetext{
* Correspondence: foster@neb.com

'Parasitology Division, New England Biolabs, 240 County Road, Ipswich, MA 01938, USA

Full list of author information is available at the end of the article
}

has consistently failed to identify these endobacteria $[8,9]$, with the exception of an intriguing indication of a Wolbachia-like endosymbiont in the plant-parasitic Tylenchid nematode Radopholus similis [10,11]. However, apart from sampling within the Onchocercidae where Wolbachia are well known, there has been almost no screening of closely related families within the superfamily Filarioidea or within sister superfamilies within the order Spirurida.

Dracunculus medinensis is a member of the superfamily Dracunculoidea, a taxon closely related to the Filarioidea within the order Spirurida. Members of these two superfamilies have similar general morphology, are all tissue dwelling parasites, ovoviviparous, and use arthropod intermediate hosts. For these reasons, the two taxa are frequently discussed as a broader filarial nematode group [12]. However, Dracunculus species can be distinguished from true filarial nematodes by certain morphological 
features, molecular phylogenies and life cycle differences. For example, unlike filarial nematodes, the first stage larvae of Dracunculus species are expelled into the environment from where they can be ingested by non-haematophagous intermediate hosts (copepods). Infection of the mammalian host occurs after ingestion of the copepod and migration of third stage larvae through the intestinal wall as opposed to filarial transmission via blood feeding of the arthropod host. D. medinensis, perhaps the longest nematode infecting humans, was until recently a major cause of human morbidity infecting $\sim 3.5$ million individuals in Africa and Asia. Despite tremendous progress towards eradication of D. medinensis by the global Guinea Worm Eradication Program, dracunculiasis persists in 4 African nations due, in part, to resource limitations, political instability and civil war [13-17]. Although prospects for complete eradication remain promising, positive identification of Wolbachia endobacteria in D. medinensis would offer another reagent in the elimination toolbox. Targeting Wolbachia with doxycycline is validated as a control method for human filariasis and is a therapy particularly well suited to individual treatment rather than mass drug administration. Delivering doxycycline to the $\sim 500$ remaining dracunculiasis patients would be a realistic goal and assist in containment of the disease since a long-lasting sterility of filarial nematodes is an early consequence of antibiotic treatment [3].

We undertook screening for the possible presence of Wolbachia in the human pathogen $D$. medinensis and in $D$. lutrae and $D$. insignis recovered from wildlife since they are representatives of the Dracunculoidea, a taxonomic group for which no information on the occurrence of Wolbachia endosymbionts is available.

\section{Parasite specimens and DNA extraction}

Three sections of female $D$. medinensis obtained from different specimens recovered from human infections in Ghana were provided by Dr Mark Eberhard, Centers for Disease Control and Prevention, Athens, GA. Specimens of D. lutrae and D. insignis were recovered from otter (Lontra canadensis) and mink (Neovison vison), respectively in Ontario, Canada as described [18]. The parasite material was stored in ethanol at $-20^{\circ} \mathrm{C}$ prior to extraction of genomic DNA by standard procedures.

\section{PCR and DNA sequencing}

The suitability of the extracted gDNA for PCR was examined by attempted amplification of part of the nematode $18 S$ rRNA gene. Primers Drac18Sf 5'-ACTGGAGGAG GAATCCAACGTGCTATGT-3' and Drac18Sr 5'-TGT GTACAAAGGGCAGGGACGTAA-3' were designed based on $18 \mathrm{~S}$ rRNA sequences of $D$. medinensis, $D$. lutrae and D. insignis [GenBank: AY947720, GenBank: JF934737, GenBank: AY947719]. PCR reactions $(25 \mu \mathrm{l})$ used Q5 High-Fidelity 2X Master Mix (New England Biolabs) with $0.5 \mu \mathrm{M}$ each primer and $\sim 50 \mathrm{ng}$ DNA. Cycling consisted of one cycle of $98^{\circ} \mathrm{C}$ for $1.5 \mathrm{~min}$, followed by 30 cycles of $98^{\circ} \mathrm{C}, 10 \mathrm{~s} ; 71^{\circ} \mathrm{C}, 20 \mathrm{~s} ; 72^{\circ} \mathrm{C}, 20 \mathrm{~s}$, then a final extension at $72^{\circ} \mathrm{C}$ for $2 \mathrm{~min}$. The PCR products were cloned into the SmaI site of pUC19 (New England Biolabs) and sequenced on both strands using a 3730xl DNA Analyzer (Applied Biosystems). Identical 166 bp sequences were obtained which matched exactly the $18 \mathrm{~S}$ rRNA sequences from these 3 species available in the NCBI database. Additional Barcode of Life primers for cytochrome c oxidase subunit 1 (COI) [19] were redesigned based on the $D$. medinensis mitochondrial genome sequence [GenBank: JN555591]. PCR, cloning and sequencing were as described for $18 \mathrm{~S}$ rRNA, but PCR used primers DracCOIf 5'-AAAGGACTAATCATAAG GATATTGG-3' and DracCOIr 5'-TAAACCTCAGGAT GACCAAAAAATCA-3' and a $61^{\circ} \mathrm{C}$ annealing temperature. Distinct 655 bp sequences were obtained from each species, which matched respective COI sequences in the NCBI database [GenBank: EU646545, GenBank: EU646601, GenBank: HQ216219] at $\geq 99 \%$ nucleotide identity. This confirmed the correct identification of each Dracunculus species, which was necessary since both $D$. insignis and D. lutrae infect the otter [18].

The possible presence of Wolbachia was examined by screening for the Wolbachia 16S rRNA gene and the genes encoding FtsZ and Wsp (Wolbachia surface protein). These genes are routinely used for Wolbachia identification and phylogenetic analyses [5,20,21]. The primers for 16S rRNA (16SwolbF, 16SwolbR3), fts $Z$ (ftsZ357F, ftsZ788R) and wsp (wsp81F, wsp691R) have been detailed elsewhere [22]. PCR was performed as described above for nematode $18 \mathrm{~S}$ rRNA except that the annealing temperatures were $57^{\circ} \mathrm{C}$ for $w s p$ and $61^{\circ} \mathrm{C}$ for $16 \mathrm{~S}$ rRNA and fts $Z$. No PCR products were obtained from any of the Dracunculus DNA samples when attempting amplification of these three Wolbachia sequences. In contrast, the correct amplicons were generated from $B$. malayi DNA (data not shown).

\section{Whole mount fluorescence staining}

Portions of female worms of all three Dracunculus species were examined for Wolbachia presence by whole mount fluorescence staining. Female $B$. malayi were used as a positive control. Worms were fixed, treated with RNase A (Sigma) at $10 \mathrm{mg} / \mathrm{ml}$ in PBS, and their DNA stained using propidium iodide (Molecular Probes), then subsequently imaged as described previously [23]. Wolbachia were not detected in either the lateral cords or embryos/microfilariae of any Dracunculus species (Figure 1). A few punctate dots of staining were observed in the lateral cord region of D. medinensis (Figure 1, Panels A, A') but these were approximately $4 \mu \mathrm{m}$ in diameter and, 
therefore, considerably larger than Wolbachia. We believe these are nuclei of nematode cells in tissue lying beneath the hypodermis. In contrast, abundant Wolbachia were observed in both the cords and embryos of $B$. malayi (Figure 1, Panels D, D').

\section{Immunohistochemistry}

Segments of female $D$. medinensis were prepared for sectioning and immunohistochemistry using a rabbit polyclonal antiserum raised to WSP as described previously [22]. B. malayi served as a positive control and tetracyclinetreated B. malayi (Wolbachia-depleted) were used in comparison to show the specificity of the anti-WSP serum. Sections of $D$. medinensis consistently showed no anti-WSP staining (Figure 2). In contrast, abundant staining was observed in the lateral cords of the B. malayi positive control. Staining in B. malayi was not observed when worms previously exposed to a 6 week tetracycline treatment to deplete their endosymbionts were examined. This differential staining of tetracycline-treated and untreated $B$. malayi proved that the positive staining is due to Wolbachia.

In conclusion, we screened the human parasitic nematode $D$. medinensis as well as D. lutrae and D. insignis recovered from Canadian wildlife for the presence of Wolbachia endosymbionts. All three species were negative for each of three Wolbachia sequences screened for by PCR using primer pairs previously used for Wolbachia screening of filarial nematodes $[5,21,22,24]$. Notably, the

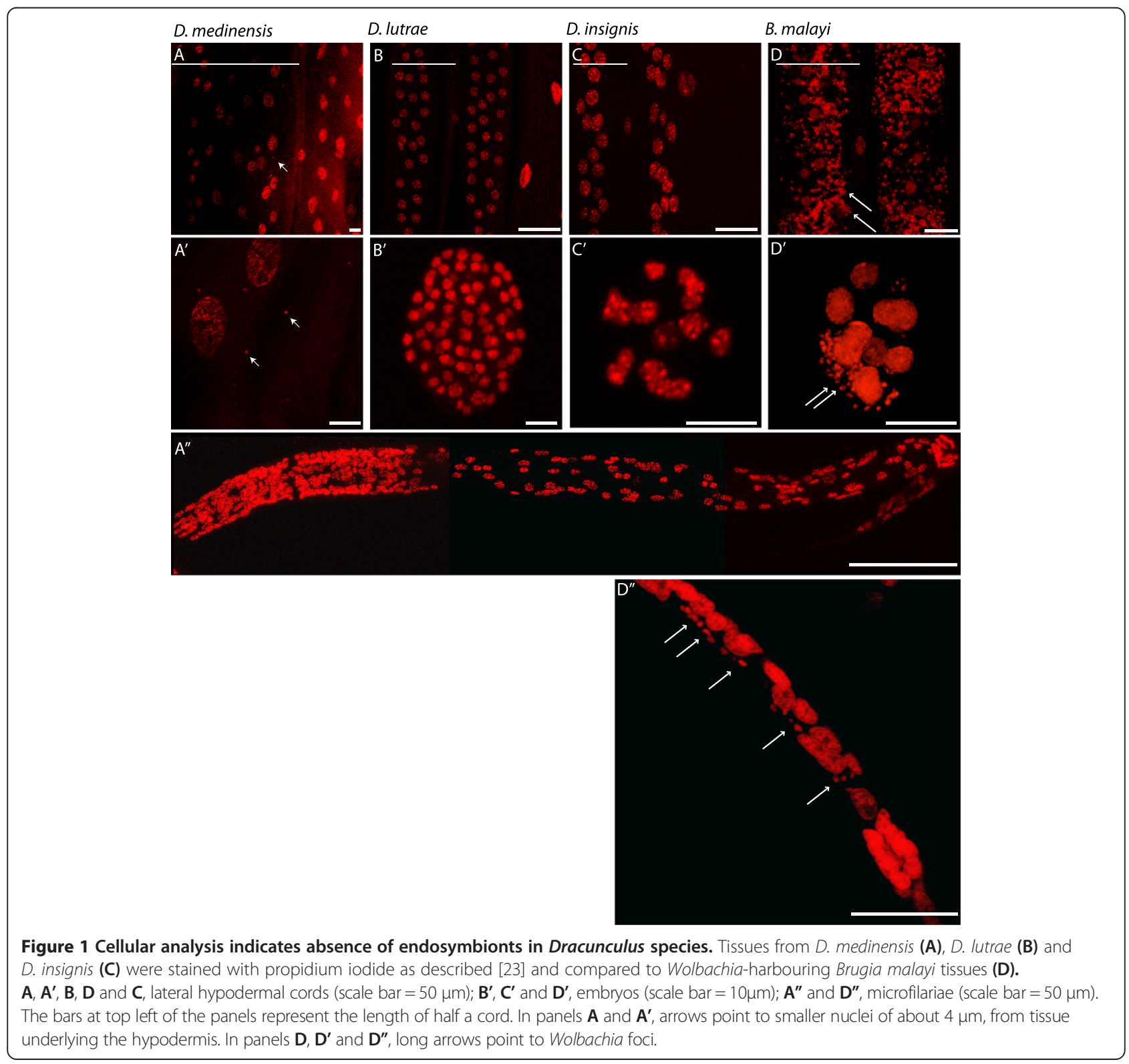




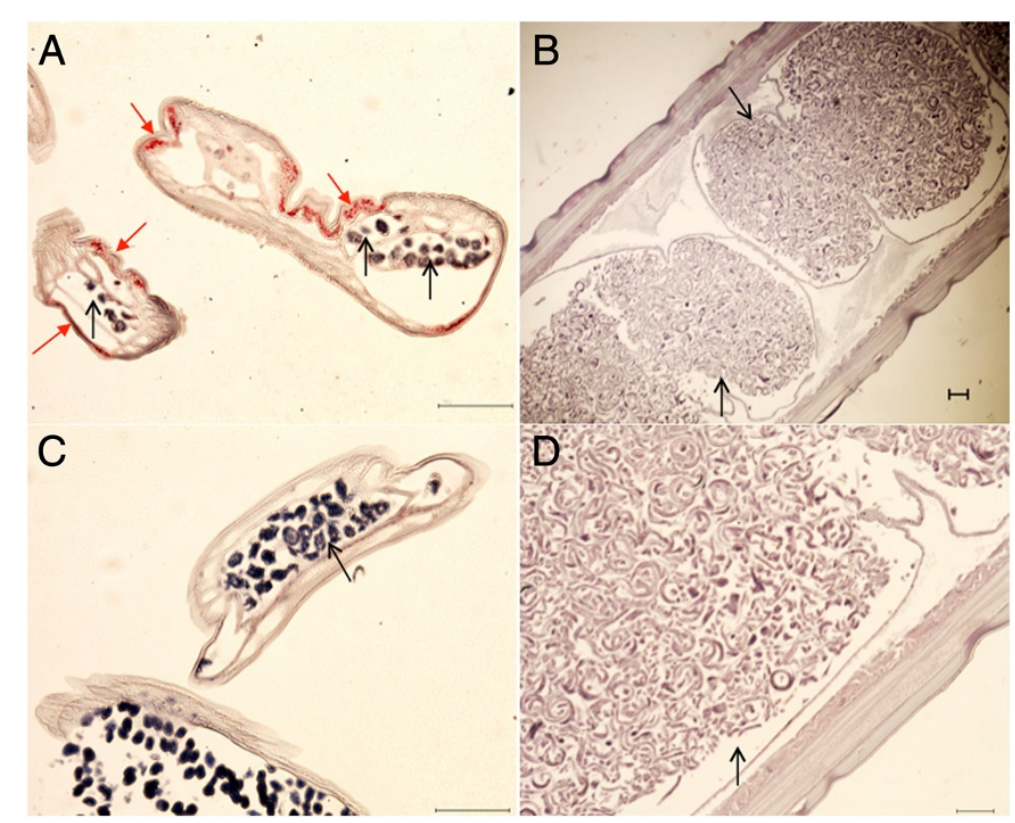

Figure 2 Anti-WSP staining in transverse sections of $D$. medinensis and B. malayi. Wolbachia are shown as red punctate staining in the lateral hypodermal cords of B. malayi (A) but are absent from D. medinensis (B and D) as well as in tetracycline-treated B. malayi (C). Solid red arrows highlight Wolbachia within the hypodermal cords. Open black arrows indicate uteri containing developing embryos. B. malayi images are ×200 magnification. D. medinensis images are $\times 40$ (B) and x100 magnification (D). Scale bars $=100 \mu \mathrm{m}$.

$w s p$ and $16 \mathrm{~S}$ rRNA primer pairs have previously been used to amplify corresponding sequences from more divergent Wolbachia strains that infect arthropods [24,25], implying that our inability to amplify from Dracunculus samples was not due to sequence divergence. Amplification of Dracunculus $18 \mathrm{~S}$ rRNA and COI sequences demonstrated the suitability of the DNA preparations for use in PCR and confirmed correct identity of the three species.

We were unable to detect Wolbachia in the three Dracunculus species by whole mount fluorescence staining, or in D. medinensis by immunohistochemistry using an anti-WSP serum that cross-reacts with Wolbachia from diverse filarial nematodes and Aedes albopictus mosquitos $[2,26,27]$. In contrast, in all experiments, our positive control B. malayi gave clear evidence of Wolbachia infection. The correct PCR amplicons were generated from B. malayi DNA and Wolbachia were readily visualized by both whole mount fluorescence and immunohistochemistry, indicating that the reagents and methodologies used were appropriate.

Our finding that three species of Dracunculus lack detectable Wolbachia supports that all members of the genus lack these endobacteria and precludes use of doxycycline as an additional tool in the efforts to finally eradicate human dracunculiasis. This is the first report of Wolbachia screening in the superfamily Dracunculoidea. The apparent lack of Wolbachia infection in members of this taxon is consistent with the notion that within the order Spirurida these endosymbionts are restricted to the superfamily Filarioidea and, more specifically, the family Onchocercidae [5,7].

\section{Competing interests}

The authors declare that they have no competing interests.

\section{Authors' contributions}

JF, MT and BS conceived and designed the study and wrote the manuscript. SE and AS collected samples and identified species. JF, FL, LF and KJ performed the experiments. FL, LF and KJ generated the figures. All authors contributed text and read and approved the final manuscript.

\section{Acknowledgements}

For financial support we thank New England Biolabs and the Bill and Melinda Gates Foundation for support of the A-WOL consortium through their grant to the Liverpool School of Tropical Medicine. We thank Dr. Don Comb for his continued interest and support.

\section{Author details}

${ }^{1}$ Parasitology Division, New England Biolabs, 240 County Road, Ipswich, MA 01938, USA. ${ }^{2}$ Centre de Biochimie Macromoléculaire, CNRS, 1919 route de Mende, 34293 Montpellier Cedex 5, France. ${ }^{3}$ Department of Parasitology, Liverpool School of Tropical Medicine, Pembroke Place, Liverpool L3 5QA, UK. ${ }^{4}$ Department of Biology, Laurentian University, 935 Ramsey Lake Road, Sudbury, ON P3E 2C6, Canada.

Received: 23 December 2013 Accepted: 20 March 2014

Published: 31 March 2014

\section{References}

1. Foster JM, Hoerauf A, Slatko BE, Taylor MJ: The Wolbachia Bacterial Endosymbionts of Filarial Nematodes. In Parasitic Nematodes Molecular Biology, Biochemistry and Immunology. 2nd edition. Edited by Kennedy MW, Harnett W. Wallingford: CABI; 2013:308-336. 
2. McGarry HF, Pfarr K, Egerton G, Hoerauf A, Akue JP, Enyong P, Wanji S, Klager SL, Bianco AE, Beeching NJ, Taylor MJ: Evidence against Wolbachia symbiosis in Loa loa. Filaria J 2003, 2:9

3. Taylor MJ, Hoerauf A, Bockarie M: Lymphatic filariasis and onchocerciasis. Lancet 2010, 376:1175-1185.

4. Lefoulon E, Gavotte L, Junker K, Barbuto M, Uni S, Landmann F, Laaksonen S, Saari S, Nikander S, de Souza Lima S, Casiraghi M, Bain O, Martin C: A new type F Wolbachia from splendidofilariinae (Onchocercidae) supports the recent emergence of this supergroup. Int J Parasitol 2012, 42:1025-1036.

5. Casiraghi M, Bain O, Guerrero R, Martin C, Pocacqua V, Gardner SL Franceschi A, Bandi C: Mapping the presence of Wolbachia pipientis on the phylogeny of filarial nematodes: evidence for symbiont loss during evolution. Int J Parasitol 2004, 34:191-203.

6. Ferri E, Bain O, Barbuto M, Martin C, Lo N, Uni S, Landmann F, Baccei SG, Guerrero R, de Souza Lima S, Bandi C, Wanji S, Diagne M, Casiraghi M: New insights into the evolution of Wolbachia infections in filarial nematodes inferred from a large range of screened species. PLoS One 2011, 6:e20843.

7. Taylor MJ, Bandi C, Hoerauf A: Wolbachia bacterial endosymbionts of filarial nematodes. Adv Parasitol 2005, 60:245-284.

8. Bordenstein SR, Fitch DH, Werren $\mathrm{JH}$ : Absence of Wolbachia in nonfilariid nematodes. J Nematol 2003, 35:266-270.

9. Duron O, Gavotte L: Absence of Wolbachia in nonfilariid worms parasitizing arthropods. Curr Microbiol 2007, 55:193-197.

10. Haegeman A, Vanholme B, Jacob J, Vandekerckhove T, Claeys M, Borgonie G, Gheysen G: An endosymbiotic bacterium in a plant-parasitic nematode: member of a new Wolbachia supergroup. Int J Parasitol 2009, 39:1045-1054.

11. Jacob J, Mitreva M, Vanholme B, Gheysen G: Exploring the transcriptome of the burrowing nematode Radopholus similis. Mol Genet Genomics 2008, 280:1-17.

12. Hotez PJ: Forgotten People, Forgotten Diseases. 2nd edition. Washington DC: ASM Press; 2013

13. Awofeso N: Towards global Guinea worm eradication in 2015: the experience of South Sudan. Int J Infect Dis 2013, 17:e577-e582.

14. Cairncross S, Tayeh A, Korkor AS: Why is dracunculiasis eradication taking so long? Trends Parasitol 2012, 28:225-230.

15. CDC: Progress toward global eradication of dracunculiasis, January 2011-June 2012. MMWR 2012, 61:854-857.

16. Hopkins DR, Ruiz-Tiben E, Weiss A, Withers PC Jr, Eberhard ML, Roy SL: Dracunculiasis eradication: and now, South Sudan. Am J Trop Med Hyg 2013, 89:5-10.

17. Visser BJ: Dracunculiasis eradication-finishing the job before surprises arise. Asian Pac J Trop Med 2012, 5:505-510.

18. Elsasser SC, Floyd R, Hebert PD, Schulte-Hostedde Al: Species identification of North American guinea worms (Nematoda: Dracunculus) with DNA barcoding. Mol Ecol Resour 2009, 9:707-712.

19. Folmer O, Black M, Hoeh W, Lutz R, Vrijenhoek R: DNA primers for amplification of mitochondrial cytochrome $c$ oxidase subunit I from diverse metazoan invertebrates. Mol Mar Biol Biotechnol 1994, 3:294-299.

20. Bandi C, Anderson TJ, Genchi C, Blaxter ML: Phylogeny of Wolbachia in filarial nematodes. Proc Bio/ Sci 1998, 265:2407-2413.

21. Bazzocchi C, Jamnongluk W, O'Neill SL, Anderson TJ, Genchi C, Bandi C: wsp gene sequences from the Wolbachia of filarial nematodes. Curr Microbiol 2000, 41:96-100.

22. Foster JM, Kumar S, Ford L, Johnston KL, Ben R, Graeff-Teixeira C, Taylor MJ: Absence of Wolbachia endobacteria in the non-filariid nematodes Angiostrongylus cantonensis and A. costaricensis. Parasit Vectors 2008, 1:31.

23. Landmann F, Foster JM, Slatko B, Sullivan W: Asymmetric Wolbachia segregation during early Brugia malayi embryogenesis determines its distribution in adult host tissues. PLOS Negl Trop Dis 2010, 4:e758.

24. Casiraghi M, Anderson TJ, Bandi C, Bazzocchi C, Genchi C: A phylogenetic analysis of filarial nematodes: comparison with the phylogeny of Wolbachia endosymbionts. Parasitology 2001, 122:93-103.

25. Zhou W, Rousset F, O'Neil S: Phylogeny and PCR-based classification of Wolbachia strains using wsp gene sequences. Proc Biol Sci 1998, 265:509-515.
26. McGarry HF, Egerton GL, Taylor MJ: Population dynamics of Wolbachia bacterial endosymbionts in Brugia malayi. Mol Biochem Parasitol 2004, 135:57-67.

27. Turner JD, Langley RS, Johnston KL, Egerton G, Wanji S, Taylor MJ: Wolbachia endosymbiotic bacteria of Brugia malayi mediate macrophage tolerance to TLR- and CD40-specific stimuli in a MyD88/ TLR2-dependent manner. J Immunol 2006, 177:1240-1249.

doi:10.1186/1756-3305-7-140

Cite this article as: Foster et al:: Absence of Wolbachia endobacteria in the human parasitic nematode Dracunculus medinensis and two related Dracunculus species infecting wildlife. Parasites \& Vectors 2014 7:140.

\section{Submit your next manuscript to BioMed Central and take full advantage of:}

- Convenient online submission

- Thorough peer review

- No space constraints or color figure charges

- Immediate publication on acceptance

- Inclusion in PubMed, CAS, Scopus and Google Scholar

- Research which is freely available for redistribution

Submit your manuscript at www.biomedcentral.com/submit
C) BioMed Central 